\title{
INVARIANT LAGRANGIAN SUBSPACES
}

\author{
LARS ANDERSSON
}

(Communicated by John B. Conway)

\begin{abstract}
It is proved that on Hilbert spaces with strong symplectic form, every symplectic operator $I+C$ with $C$ compact has an invariant Lagrangian subspace.
\end{abstract}

1. Introduction. It is a well-known fact that every symplectic operator on a finite-dimensional complex vector space has a Lagrangian invariant subspace. In this note we generalize the result on invariant Lagrangian subspaces to symplectic operators of the form $I+C$ with $C$ compact on a Hilbert space with strong symplectic form. The proof uses a method due to Arveson and Feldman [1].

The relation between Lagrangian subspaces and complex structures is of importance in the quantization problem [8]. The result on existence of invariant Lagrangian subspaces proved here is a step towards extending the results of Paneitz [4] which assume a bound on $C$.

Let $(H,\langle\rangle$,$) be a separable Hilbert space over the field \mathbb{C}$ equipped with a strong symplectic form $\omega$, i.e. a skew-symmetric continuous bilinear form such that the induced mapping $\hat{\omega}: H \rightarrow H^{*}$ defined by $\langle\hat{\omega} x, y\rangle=\omega(x, y)$ is an isomorphism.

Let $L(H)$ denote the space of continuous linear automorphisms of $H$. An element $A \in L(H)$ is said to be symplectic if $\omega(A x, A y)=\omega(x, y), \forall x, y \in H$ and the subgroup of $\mathrm{GL}(H)$ consisting of symplectic operators with respect to $\omega$ is denoted by $\operatorname{Sp}(H, \omega)$. The subgroup of $\operatorname{Sp}(H, \omega)$ consisting of operators of the form $(I+C)$ with $C$ compact is denoted by $\operatorname{Sp}_{C}(H, \omega)$ and the corresponding Lie algebra by $\mathbf{s p}_{C}(H, \omega)$.

Let $L \subset H$ be a linear subspace. Then we define the $\omega$-orthogonal complement $L^{\perp}$ of $L$ by

$$
L^{\perp}=\{x \in H \mid \omega(x, y)=0, \forall y \in L\} .
$$

A subspace $L \subset H$ is said to be isotropic if $L \subset L^{\perp}$, coisotropic if $L^{\perp} \subset L$ and symplectic if $L \cap L^{\perp}=\{0\}$. A closed isotropic subspace $L$ is said to be Lagrangian if there exists another closed isotropic subspace $L^{\prime}$ such that $H=L \oplus L^{\prime}$.

The proof of $[\mathbf{7}$, Proposition 5.1] extends easily to Hilbert spaces over $\mathbb{C}$ to show that a maximal isotropic subspace (i.e. $L=L^{\perp}$ ) is Lagrangian in the present case.

We are now ready to state our main theorem.

Received by the editors December 3, 1985 and, in revised form, October 27, 1986 and April 16, 1987.

1980 Mathematics Subject Classification (1985 Revision). Primary 47A15; Secondary 81C25.

Key words and phrases. Invariant subspace, symplectic transformation.

The author was supported by a Post-Doctoral scholarship from the Swedish Natural Sciences Research Council. 
THEOREM 1. Let $(H, \omega)$ be as above. Then, for any $A \in \operatorname{Sp}_{C}(H, \omega)$, there exists a Lagrangian subspace $L \subset H$ which is invariant under $A$, i.e. $A L \subset L$. The same statement holds for $X \in \operatorname{sp}_{C}(H, \omega)$.

Theorem 1 will be proved in $\S 2$. In the corollaries below we record some facts that can be proved using Theorem 1 . It is also possible to prove these directly using finite-dimensional approximations of $C$ and the continuity of spectral data.

Let $C$ be a compact operator on $H$. Then the spectrum of $C$ consists of an at most countable number of points with 0 as their only point of accumulation. The nonzero points in the spectrum are always eigenvalues of finite multiplicity, but the point 0 which is always in the spectrum can be "infinitely degenerate" and indeed, a compact operator may lack eigenvalues. See [5] for discussion and examples. To study the properties of the spectrum of symplectic operators in $\mathrm{Sp}_{C}$ we use the following normal form, which is a standard result on symplectic operators with an invariant Lagrangian subspace; see [6, Proposition 2.5] for the real case.

Proposition 1. Let $(H, \omega)$ be as above, let $A \in \operatorname{Sp}(H, \omega)$ and assume that $A$ has an invariant Lagrangian subspace $L$. Then there is a symplectomorphism $\rho:(H, \omega) \rightarrow\left(L \oplus L^{*}, \Omega\right)$, where $\Omega$ is the canonical symplectic form on $L \oplus L^{*}$, which transforms $A$ to the form

$$
\rho \circ A \circ \rho^{-1}=\left[\begin{array}{cc}
B & B E \\
0 & \left(B^{*}\right)^{-1}
\end{array}\right],
$$

where $B: L \rightarrow L$ is invertible and $E: L^{*} \rightarrow L$ defines a symmetric bilinear form on $L^{*}$, which in this case is bounded. For $X \in \mathbf{s p}(H, \omega)$ the corresponding form is

$$
\rho \circ X \circ \rho^{-1}=\left[\begin{array}{cc}
Z & E \\
0 & -Z^{*}
\end{array}\right],
$$

where $Z$ is any bounded operator and $E$ is as above.

This normal form immediately gives the following characterization of the spectrum of symplectic operators in $\mathrm{Sp}_{C}$.

COROLlaRY 1. Let $(H, \omega)$ be a symplectic Hilbert space over $\mathbf{R}$ with strong symplectic form $\omega$ and let $A \in \operatorname{Sp}_{C}(H, \omega)$. Let $\lambda$ be in the spectrum of $A$. Then $\lambda, \bar{\lambda}, \lambda^{-1} \bar{\lambda}^{-1}$ all have the same multiplicity. In particular, the multiplicity of 1 is even or infinite.

For $X \in \operatorname{sp}_{C}(H, \omega)$, the corresponding statement is that if $\lambda$ is in the spectrum of $X$, then $\lambda, \bar{\lambda},-\lambda,-\bar{\lambda}$ all have the same multiplicity.

ProOF. Extend $A$ and $\omega$ to the complex Hilbert space $H \otimes \mathbb{C}$. Then Theorem 1 gives an invariant Lagrangian subspace so we can apply Proposition 1 . The result now follows from standard spectral theory.

Let $\operatorname{Sp}_{r}(H, \omega)$ (with Lie-algebra $\mathbf{s p}_{r}(H, \omega)$ ) denote the space of symplectic operators of the form $A=I+C$ with $C \in L^{r}(H)$, where, for $r=1, L^{1}$ denotes the space of traceclass operators on $H$ and for $r=0, L^{0}$ denotes the space of operators on $H$ with finite rank.

For an operator of the form $A=I+C$ with $C \in L^{1}(H)$,

$$
\operatorname{det}(A)=\prod_{i=1}^{N(A)} \lambda_{i}
$$


where $\operatorname{det}(A)$ denotes the Fredholm determinant of $A, \lambda_{i}(A)$ are its eigenvalues, with multiplicity $N(A)$ (see [5]). Similarly, for $X \in L^{1}(H)$,

$$
\operatorname{tr}(X)=\sum_{i=1}^{N(X)} \lambda_{i}(X)
$$

Applying this to the result in Corollary 1 gives

Corollary 2. Let $A \in \operatorname{Sp}_{1}(H, \omega)$. Then $\operatorname{det}(A)=1$. Let $X \in \mathbf{s p}_{1}(H, \omega)$. Then $\operatorname{tr}(X)=0$.

2. Proof of Theorem 1. We will need a few preliminaries.

LEMMA 1. Let $A \in \operatorname{Sp}_{C}(H, \omega)$ and let $L \subset H$ be a closed invariant subspace of $A$. Then $L^{\perp}$ is a closed invariant subspace of $A$.

Proof. Since $A \in \mathrm{Sp}_{C},\left.A\right|_{L}: L \rightarrow L$ is injective and has index zero and hence is surjective. Thus, every $y \in L$ satisfies $y=A x$ for some $x \in L$ which implies $A^{-1} y=A^{-1} A x=x \in L$. This shows that $A^{-1} L=L$. Let now $x \in L^{\perp}$ and note that $\omega(A x, y)=\omega\left(x, A^{-1} y\right)=0$ for all $y \in L$. It follows by the definition of $L^{\perp}$ that $A x \in L^{\perp}$.

LEMMA 2. Let $L \subset H$ be an isotropic subspace. Then $L^{\perp} / L$ has an induced symplectic form $\omega^{\prime}$. Let $W \subset L^{\perp} / L$ be an isotropic subspace with respect to $\omega^{\prime}$. Then there is a unique subspace $W_{0}$ of $L^{\perp}$ such that $W_{0} / L=W$. Further, $W_{0}$ is isotropic.

ProOF. This is standard, except for the last statement. Let $W_{0}$ be the (unique) preimage of $W$ under the projection $\pi: L^{\perp} \rightarrow L^{\perp} / L$. Then, for $x, y \in W^{0}$ we have $\omega(x, y)=\omega^{\prime}(\pi(x), \pi(y))=0$.

LEMMA 3. Let $A \in \mathrm{Sp}_{0}(H, \omega)$. Then $A$ has an invariant Lagrangian subspace.

REMARK. The proof of Lemma 3 is a straightforward extension of the proof for the finite-dimensional case (see [2]) but is included since it is used as a model for the final proof.

PROOF. We can use the same method as in the finite-dimensional case, since $A \in \operatorname{Sp}_{0}(H, \omega)$ implies that $A$ has a complete set of generalized eigenvectors. Thus, let $e \in H$ be an eigenvector of $A$ and let $L$ denote the span of $e$. Then $L$ is an invariant isotropic subspace for $A$.

Consider the set of all nontrivial invariant isotropic subspaces for $A$. By Zorn's Lemma, this has a maximal element which we denote by $L$. We claim that $L$ is also maximal isotropic in $H$ and hence Lagrangian by [7, Proposition 5.1].

To prove this, we assume the contrary, i.e. that $L$ is not maximal isotropic in $H$. In particular, this means that $L^{\perp} / L$ is nontrivial. Note that $L^{\perp}$ is also invariant by Lemma 1 so we get an induced operator $A_{L^{\perp} / L}$ on $L^{\perp} / L$. Clearly, $A_{L^{\perp} / L} \in \mathrm{Sp}_{0}\left(L^{\perp} / L, \omega_{L^{\perp} / L}\right)$ so we again find an eigenvector which spans a one dimensional isotropic subspace $W \subset L^{\perp} / L$. Thus, we can apply Lemma 2 to get an invariant isotropic subspace $L^{\prime} \subset H$ such that $L \subset L^{\prime}$ with strict inclusion. Hence, $L$ is not maximal among the invariant isotropic subspaces for $A$, which is absurd. 
We have derived a contradiction from the assumption that a subspace which is maximal among the $A$-invariant isotropic subspaces is not maximally isotropic in $H$. This completes the proof of Lemma 3 .

Let us recall some standard material concerning Hilbert spaces with a strong symplectic form. Let $(H, \omega)$ be as above and let a Lagrangian subspace $W \subset H$ be given. Then (see [2]) there is a symplectomorphism $\rho$ between $(H, \omega)$ and $(V, \sigma)$, where $V=W \times W^{*}$ and $\sigma$ is the canonical symplectic structure on $V$, defined as follows: Let $\left(x_{1}, x_{2}\right),\left(y_{1}, y_{2}\right) \in V=W \times W^{*}$. Then we set

$$
\sigma\left(\left(\begin{array}{l}
x_{1} \\
x_{2}
\end{array}\right),\left(\begin{array}{l}
y_{2} \\
y_{2}
\end{array}\right)\right)=\left(x_{1}, y_{2}\right)-\left(x_{2}, y_{1}\right)
$$

where the pairings are the obvious ones.

Clearly, given $(H, \omega)$ we can, by choosing a Lagrangian subspace and using the map $\rho:(H, \omega) \rightarrow(V, \sigma)$ to form the isomorphism $A \rightarrow \rho^{-1} \circ A \circ \rho: L(H) \rightarrow L(V)$ without loss of generality assume that $H=W \times W^{*}$ and that $\omega$ is the canonical symplectic structure.

Now define a linear mapping $\hat{\omega}: H \rightarrow H^{*}$ by $\omega(x, y)=\langle\hat{\omega} x, y\rangle_{H^{*}, H}$ for $x, y \in H$. In matrix form, $\hat{\omega}$ can be represented as

$$
\hat{\omega}\left(\begin{array}{l}
x_{1} \\
x_{2}
\end{array}\right)=\left[\begin{array}{cc}
0 & -I \\
I & 0
\end{array}\right]\left(\begin{array}{l}
x_{1} \\
x_{2}
\end{array}\right)=\left(\begin{array}{c}
-x_{2} \\
x_{1}
\end{array}\right) \in W^{*} \times W=H^{*}
$$

where the I's are the identity operators of $W$ and $W^{*}$. Further, define its adjoint $\hat{\omega}^{*}$ by $\langle\hat{\omega} x, y\rangle_{H^{*}, H^{*}}=\left\langle x, \hat{\omega}^{*} y\right\rangle_{H, H}$ for $x, y \in H \times H^{*}$. Using (2.1) it is straightforward to verify that $\hat{\omega}^{*}$ in matrix form is represented by (let $\left(x_{1}, x_{2}\right) \in H^{*}=W^{*} \times W$ )

$$
\hat{\omega}^{*}\left(\begin{array}{l}
x_{1} \\
x_{2}
\end{array}\right)=\left[\begin{array}{cc}
0 & I \\
-I & 0
\end{array}\right]\left(\begin{array}{l}
x_{1} \\
x_{2}
\end{array}\right)=\left(\begin{array}{c}
x_{2} \\
-x_{1}
\end{array}\right) \in W^{*} \times W=H^{*} .
$$

From this it is immediate that $\hat{\omega}$ is an isometry and that

$$
\left(\hat{\omega}^{*}\right)^{-1}=\left(\hat{\omega}^{-1}\right)^{*}=\hat{\omega}
$$

where we have defined $\left(\hat{\omega}^{-1}\right)^{*}$ analogously to $\hat{\omega}^{*}$. It is these special properties that make the following argument work.

Let $\phi: L(H) \rightarrow L(H)$ be defined by $\phi(X)=\hat{\omega}^{-1} X^{*} \hat{\omega}$ where $X^{*} \in L\left(H^{*}\right)$ is the conjugate operator to $X$, defined by $\langle X y, z\rangle_{H, H^{*}}=\left\langle y, X^{*} z\right\rangle_{H, H^{*}}$ for $(y, z) \in$ $H \times H^{*}$.

LEMMA 4. Let $H=W \times W^{*}$ and let $\omega$ be the canonical symplectic form on $H$. Let $P: H \rightarrow H$ be an orthogonal projection onto a Lagrangian subspace of $H$. Then the following statements are true.

(1) $\phi(P)$ is an orthogonal projection onto a Lagrangian subspace,

(2) $\phi(P) P=P \phi(P)=0$,

(3) $P+\phi(P)=I_{H}$, the identity operator on $H$.

PROOF. Using the assumption that $P$ is an orthogonal projection onto a Lagrangian subspace $L$ of $H$, we can again find a symplectomorphism $\rho: H \rightarrow V=$ $L \times L^{*}$. Due to the fact that $H=W \times W^{*}$ with the canonical symplectic structure $\omega$ we can take $\rho$ to be an isometry. Denote the canonical symplectic structure of $V$ by $\sigma$. Now we use $\rho$ to move our operators to $V$ :

$$
\begin{gathered}
X \rightarrow \rho_{*} X=\rho \circ X \circ \rho^{-1}: L(H) \rightarrow L(V), \\
X^{*} \rightarrow \rho_{*} X^{*}=\rho^{-*} X^{*} \rho^{*}: L\left(H^{*}\right) \rightarrow L\left(V^{*}\right) .
\end{gathered}
$$


Consider the effect of these operations on $P$ and $\phi(P)$. By the construction of $\rho$, it takes $L \times H / L \rightarrow L \times \hat{\omega}(H / L)=L \times L^{*}$, so we see that $\rho_{*} P$ becomes projection onto the first component and $\rho_{*} \phi(P)$ becomes projection onto the second component.

By the linearity of $\rho_{*}$ and the isometry properties of $\rho$, we can easily deduce the statements in the Lemma. For part (1), note that $\phi(P)$ is an orthogonal projection since $\rho_{*} \phi(P)$ is one and its range is $\rho^{-1}\left(0 \times L^{*}\right)$ which is the image of a Lagrangian subspace by a symplectomorphism and hence Lagrangian.

Part (2) follows from the selfadjointness of $P$ and $\phi(P)$ and the fact that $0=$ $\left(\rho_{*} P\right)\left(\rho_{*} \phi(P)\right)=\rho_{*}(P \phi(P))$.

The statement in part (3) follows from the fact that $\rho_{*}(P+\phi(P))=I_{V}$, the identity operator on $V$.

LEMMA 5. Let $(H, \omega)$ and $W$ be as in Lemma 4. Let $B \in L^{1}(W)$ be injective and nonnegative and assume that $\operatorname{tr}(B)=1 / 2$. Let the state $\Omega: L(H) \rightarrow \mathrm{C}$ be given by $\Omega(X)=\operatorname{tr}\left(\left(B \oplus B^{*}\right) \circ X\right)$. Then $\Omega$ is a faithful normal state on $L(H)$ and $\Omega(P)=1 / 2$ for any orthogonal projection $P$ onto a Lagrangian subspace of $(H, \omega)$.

Proof. The only statement that is not obvious is that $\Omega(P)=1 / 2$. To see this, note that by Lemma 4 ,

$$
1=\Omega(I)=\Omega(P)+\Omega(\phi(P)) .
$$

Now, using the matrix form of $\hat{\omega}$ and $\hat{\omega}^{-1}=\hat{\omega}^{*}$, we get

$$
\phi\left(B \oplus B^{*}\right)=\left[\begin{array}{cc}
0 & I \\
-I & 0
\end{array}\right]\left[\begin{array}{cc}
B^{*} & 0 \\
0 & B
\end{array}\right]\left[\begin{array}{cc}
0 & -I \\
I & 0
\end{array}\right]=\left[\begin{array}{cc}
B & 0 \\
0 & B^{*}
\end{array}\right]=B \oplus B^{*} .
$$

Thus we have $\Omega(\phi(P))=\operatorname{tr} \phi\left(\left(B \oplus B^{*}\right) \circ P\right)$. By the isometry properties of $\hat{\omega}$ we find that $\Omega(\phi(P))=\Omega(P)$, so we get $2 \Omega(P)=1$.

We now apply the above and the method of proof used in [1] to the problem of finding an invariant isotropic subspace for operators in $\operatorname{Sp}_{C}(H, \omega)$. This will allow us to generalize the method used in the proof of Lemma 3 to operators in $\mathrm{Sp}_{C}$. By the remark before Lemma 4 we can, without loss of generality assume that $H=W \times W^{*}$ and that $\omega$ is the canonical symplectic form on $H$.

LemMA 6. Let $(H, \omega)$ be as above and let $A \in \operatorname{Sp}_{C}(H, \omega)$. Then $A$ has a nontrivial invariant isotropic subspace.

PROOF. If $A$ has an eigenvector $e$, then the span of $e$ is an invariant isotropic subspace. Thus, in the following we may without loss of generality assume that $A$ has no eigenvectors.

It is well known that $\operatorname{Sp}_{C}(H, \omega)$ is the closure with respect to the norm topology of $\operatorname{Sp}_{0}(H, \omega)$ (to see this, use the surjectivity of the exponential mapping and apply the approximation property for compact operators in $\mathbf{s p}_{C}(H, \omega)$, cf. [3]). Thus, let $\left\{A_{n}\right\}_{n=1}^{\infty}$ be a sequence of operators in $\operatorname{Sp}_{0}(H, \omega)$ such that $\left\|A_{n}-A\right\|_{L(H)} \rightarrow 0$ as $n \rightarrow \infty$. Let $L_{n}$ be an invariant Lagrangian subspace for $A_{n}$, given by Lemma 3 and let $P_{n}$ be the orthogonal projection onto $L_{n}$. Let $\Omega$ be as in Lemma 5 , so that $\Omega\left(P_{n}\right) \equiv 1 / 2$.

Now let $P \in L(H)$ be an accumulation point of $\left\{P_{n}\right\}$ with respect to the weak topology and in the following, let $\left\{P_{n}\right\}$ denote a convergent subsequence. Then $\Omega(P)=1 / 2$, so $P \neq 0$ and if we let $M=\{x \mid P x=x\}$, then $M \neq H$. 
By construction, $\left(P_{n}-I\right) A_{n} P_{n}=0$ for all $n$, so

$$
\left\|\left(P_{n}-I\right) A P_{n}\right\| \leq\left\|\left(P_{n}-I\right)\left(A-A_{n}\right) P_{n}\right\|+\left\|\left(P_{n}-I\right) A_{n} P_{n}\right\| \leq\left\|A-A_{n}\right\|,
$$

which tends to 0 as $n \rightarrow \infty$. This is the crucial property used in [1]. Following the proof in $[\mathbf{1}]$ we find that for $x \in M, P_{n} x \rightarrow x$ in norm, since

$$
\left\|P_{n} x-x\right\|^{2}=\left(P_{n} x, P_{n} x\right)-2\left(P_{n} x, x\right)+(x, x)=-\left(P_{n} x, x\right)+(x, x) \rightarrow 0,
$$

where we have used the projection property and selfadjointness of $P_{n}$ It follows that

$$
\omega(x, y)=\lim _{n \rightarrow \infty} \omega\left(P_{n} x, P_{n} y\right)=0, \quad \forall x, y \in M
$$

so $M$ is an isotropic subspace of $H$. To see that $M$ is also invariant, note that for $x \in M, A P_{n} x \rightarrow A x$ in norm by (2.3), so $P_{n} A P_{n} x \rightarrow P A x$ weakly. Now, using (2.2) we can conclude that $A P_{n} x \rightarrow P A x$ weakly, but by the above, $A P_{n} x \rightarrow A x$ in norm, so $A x=P A x$ and hence $A x \in M$.

Finally, we have to check that $M$ is nontrivial. Let $C=A-I$. Then, since $A \in \operatorname{Sp}_{C}(H, \omega)$ by assumption, $C$ is compact. Let $x \in H$ be arbitrary. By the weak convergence of $P_{n} \rightarrow P$ and the compactness of $C$ it follows that $C P_{n} x \rightarrow C P x$ in norm. Again by the weak convergence of $P_{n}$ to $P$, we have that $P_{n} C P_{n} x \rightarrow P C P x$ weakly, but using (2.2) we find that $\left\|P_{n} C P_{n}-C P_{n}\right\| \rightarrow 0$, so in fact $C P_{n} x \rightarrow P C P x$ weakly. But we just proved that the left side converges in norm to $C P x$ and it follows that $C P x=P C P x$.

This completes the proof since by the remark at the beginning, we can assume that $A$ has no eigenvalues and in particular that the nullspace of $C$ is trivial. Hence by choosing $x \in H$ so that $P x \neq 0$, which is always possible since $P \neq 0, C P x$ provides a nonzero element of $M$. Thus, $M$ is a nontrivial isotropic subspace, invariant under $A$.

REMARK. Apart from using the projections $P_{n}$ onto Lagrangian subspaces, the special state $\Omega$ and noting that $M$ is isotropic, the proof of Lemma 6 follows very closely that of [1, Theorem, p. 62]. The author is grateful to Professor Arveson for pointing this paper out to him.

We are now ready to complete the proof of Theorem 1 . Let $A \in \operatorname{Sp}_{C}(H, \omega)$. By Lemma 6 , there is a nontrivial invariant isotropic subspace for $A$. Consider as in the proof of Lemma 3, the set of all invariant isotropic subspaces for $A$. By Zorn's Lemma, this set has a maximal element which we call $L$. We wish to show that $L$ is a maximal isotropic subspace of $H$ and hence Lagrangian.

To do this, assume that $L$ is not maximally isotropic in $H$. Then $L^{\perp} / L$ is a nontrivial symplectic space and arguing as in the proof of Lemma 3, we see that the induced operator $A_{L^{\perp} / L}$ is in $\operatorname{Sp}_{C}\left(L^{\perp} / L, \omega_{L^{\perp} / L}\right)$ and hence by Lemma 6 that there is a nontrivial invariant isotropic subspace for $A_{L^{\perp} / L}$. By lifting to $H$ as in the proof of Lemma 3, we find that $L$ is not maximal among the invariant isotropic subspaces for $A$, a contradiction. It follows that $L$ is maximally isotropic in $H$. The same method gives the result for $X \in \mathbf{s p}_{C}(H, \omega)$. This completes the proof of Theorem 1.

ACKNOWLedgment. The author is grateful to William Arveson and Sheldon Axler for some very helpful discussions. 


\section{REFERENCES}

1. W. B. Arveson and J. Feldman, A note on invariant subspaces, Michigan Math. J. 15 (1967), 61-64.

2. J. J. Duistermaat, Fourier integral operators, Courant Institute Lecture Note, New York Univ., 1973.

3. P. Harpe, Classical Banach-Lie algebras and Banach-Lie groups of operators in Hilbert space, Lecture Notes in Math., vol. 285, Springer-Verlag, Berlin, 1972.

4. S. M. Paneitz, Hermitian structures on solution varieties of nonlinear relativistic wave equations, Lecture Notes in Math., vol. 905, Springer-Verlag, Berlin, 1980, 108-118.

5. M. Reed and B. Simon, Analysis of operators, Methods of Modern Mathematical Physics, 4, Academic Press, New York, 1978.

6. R. C. Swanson, Linear symplectic structures on Banach spaces, Rocky Mountain J. Math. 10 (1980), 305-317.

7. A. Weinstein, Symplectic manifolds and their Lagrangian submanifolds, Adv. in Math. 6 (1971), 329-346.

8. N. M. J. Woodhouse, Geometric quantization, Clarendon Press, Oxford, 1980.

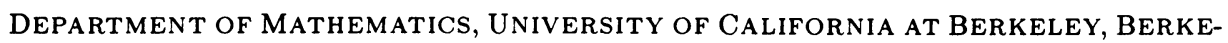
LEY, CALIFORNIA 94607

Current address: Department of Mathematics, The Royal Institute of Technology, S-100 44 Stockholm, Sweden 